\title{
Advances in the Management of Multiple Sclerosis Spasticity: Multiple Sclerosis Spasticity Nervous Pathways
}

\author{
Diego Centonze
}

UOSD Multiple Sclerosis Clinical and Research Center, Tor Vergata University \& Laboratory of Neuroimmunology and Synaptic Plasticity, CERC-Santa Lucia Foundation, Rome, Italy

\section{Key Words}

Cannabinoid receptors - Corticospinal tract injury .

Glutamatergic pathways - Motor cortex plasticity .

Stretch reflex $\cdot$ THC:CBD oromucosal spray

\begin{abstract}
Background: Spasticity arises from hyperexcitability of the neural stretch reflex arc secondary to injury of the corticospinal tract. In response to injury, the density of glutamatergic inputs from afferent $1 \mathrm{~A}$ fibers to motor neurons increases dramatically and adaptive changes occur in the morphology of microglia cells in the spinal cord. Summary: Involvement of the endocannabinoid system in pathophysiological mechanisms responsible for spasticity has been demonstrated in animal models of MS. Stimulation of cannabinoid (CB) receptors reduces the hyperglutamatergic drive from sensory afferents to spinal cord motor neurons and blocks the synaptic effects of activated microglia and pro-inflammatory mediators (e.g. TNF-a) on glutamatergic transmission. Enhancing corticospinal tract excitability through intermittent theta burst stimulation inhibits the stretch reflex and spasticity by promoting long-term potentiation, a form of synaptic plasticity that requires stimulation of $\mathrm{CB}_{1}$ receptors. Evidence indicates that the antispasticity effects of THC:CBD oromucosal spray (Sativex ${ }^{\circledR}$ ) are associated with enhanced
\end{abstract}

cortical long-term potentiation. Key Messages: Glutamatergic and GABAergic pathways are involved in the regulation of muscle tone. $\mathrm{CB}_{1}$ receptors, which are associated with movement, postural control, and pain and sensory perception, influence glutamatergic pathways. THC:CBD oromucosal spray was shown to reverse motor cortex plasticity from long-term depression through long-term potentiation of synaptic transmission, thereby restoring, at least in part, effective corticospinal inputs to spinal circuits.

두 2014 S. Karger AG, Base

\section{Pathophysiology of Muscle Tone Control and Spasticity Generation}

The most plausible mechanism at the basis of spasticity is hyperexcitability of the neural stretch reflex arc secondary to injury of the corticospinal tract [1]. The stretch reflex arc is composed mainly of afferent $1 \mathrm{~A}$ fibers activated by muscle stretch and detected at the level of muscle spindle; these fibers activate alpha motor neurons directed to the same muscle. Activation of secondary motor neurons counteracts muscle elongation by causing contraction. It is generally accepted that the corticospinal tract has inhibitory control of this mechanism.

\section{KARGER}

E-Mail karger@karger.com

www.karger.com/ene
(C) 2014 S. Karger AG, Basel

0014-3022/14/0721-0006\$39.50/0
Dr. D. Centonze

UOSD Multiple Sclerosis Clinical and Research Center

Department of Systems Medicine, Tor Vergata University

Via Montpellier 1, IT-00133 Rome (Italy)

E-Mail centonze@uniroma2.it 
The pathophysiological mechanism of stretch reflex arc hyperexcitability induced by selective injury has been investigated at the level of single neuron morphology. In response to corticospinal tract injury, the density of glutamatergic inputs from afferent $1 \mathrm{~A}$ fibers to motor neurons increases dramatically and adaptive changes occur in the morphology of microglia cells in the spinal cord [2].

Given the relevance of reactive microgliosis in the pathophysiology of MS, its possible role in the regulation of excitatory synaptic inputs to central neurons was addressed. In the presence of microglial cells derived from primary cultures of immortalized cell lines, the shape and duration of spontaneous excitatory postsynaptic currents (sEPSCs), a neurophysiological measure of glutamatergic inputs to motor neurons, was increased [3]. Inhibition of this effect by etanercept, a tumour necrosis factor (TNF)- $\alpha$ antagonist, indicated that microgliosis modulates glutamatergic transmission contributing to hyper-excitability of corticospinal reflexes through the release of pro-inflammatory mediators.

\section{Role of the Endocannabinoid System in Control of MS Spasticity}

The role of the endocannabinoid system in controlling spasticity has been demonstrated in various animal models of MS.

In mice with chronic relapsing experimental allergic encephalomyelitis (CREAE), modulating the endogenous tone of endocannabinoids through various pharmacological manipulations that inhibited the synthesis, transport or degradation of anandamine reduced the resistance to flexion; this effect was inhibited in the presence of $\mathrm{CB}_{1}$ and $\mathrm{CB}_{2}$ receptor antagonists [4]. In this same mouse model of MS, THC + CBD at a 1:1 ratio (equivalent to Sative ${ }^{\circledR}$ ) reduced spasticity in a dose dependent manner; the effect at a dose of $10 \mathrm{mg} / \mathrm{kg}$ was similar to that of baclofen $5 \mathrm{mg} / \mathrm{kg}$ [5].

In chick embryo spinal cords, stimulation of $\mathrm{CB}_{1}$ receptors with the specific cannabinoid agonist WIN inhibited the frequency of sEPSCs by reducing glutamatergic inputs to alpha motor neurons, but had no effect on GABAergic inputs, indicating that cannabinoids ameliorate spasticity by selectively reducing the hyperglutamatergic drive from sensory afferents to spinal cord motor neurons [6]. Elsewhere, Rossi and colleagues demonstrated in CREAE mice that stimulation of $\mathrm{CB}_{1}$ receptors with the cannabinoid agonist HU201 attenuated alteration in the shape and duration of striatal glutamate-mediated sEPSCs by TNF- $\alpha$ [7].

Multiple Sclerosis Spasticity Nervous Pathways
Collectively, the results of these studies confirm the role of the endocannabinoid system in pathophysiological mechanisms responsible for the generation of spasticity.

Another interesting means of controlling spasticity involves modulation of plasticity at the level of the motor cortex. Repetitive transcranial magnetic stimulation (rTMS) induces adaptations in cortical neuronal circuitries. Application of rTMS over the leg motor corticol area in 10 human volunteers reduced activation of the stretch reflex by increasing corticospinal drive to the spinal cord in specific spinal circuitries [8]. A refinement of this technique known as theta burst stimulation (TBS) induces long-term potentiation of synaptic transmission in the motor cortex after only a short application period (90120 seconds) [9]. In a study involving $20 \mathrm{MS}$ patients with lower limb spasticity, the group who received 5 sessions of rTMS in the form of intermittent TBS showed a significant and persisting reduction in the stretch reflex (measured by H/M amplitude ratio) and improvement in modified Ashworth Scale scores compared with the group who received sham stimulation [10]. To explore the potential involvement of the cannabinoid system on TBSinduced synaptic plasticity in humans, the effects of THC:CBD oromucosal spray on continuous TBS and intermittent TBS protocols were tested in patients with MS [11]. Whereas continuous TBS induced inhibition of motor-evoked potentials (MEP) before exposure to THC:CBD oromucosal spray, it caused persisting enhancement of MEP amplitude at 4 weeks after exposure, implicating the cannabinoid system in the modulation of synaptic plasticity.

Confirmation that cannabinoids are involved in ameliorating spasticity, not only by regulating spinal cord circuits, but by regulating the excitability of the motor cortex, has come from a recent observation in a group of MS patients who had genetic variants associated with reduced expression of cannabinoid receptors [12]. Individuals with a long number of repeats in the promoter region of cannabinoid receptors had reduced $\mathrm{CB}_{1}$ receptor protein expression in peripheral tissues, possibly indicative of a defective cannabinoid receptor mechanism. A particularly intriguing study result was the association between a 'long AAT' phenotype and poor clinical response to rehabilitation. Whereas a classical rehabilitation protocol produced some improvement in Expanded Disability Status Scale and spasticity 0-10 Numerical Rating Scale scores in patients with a preserved genetic cannabinoid signal, there was no effect in those with altered cannabinoid regulation mechanisms [manuscript submitted]. 


\section{Disclosures/Conflict of Interest}

DC has received research grants and/or honoraria from Almirall, Bayer Schering, Biogen Idec, Genzyme, GW Pharmaceuticals, Merck Serono, Novartis, Sanofi-Aventis and Teva.
Writing assistance was provided by Content Ed Net (Madrid, Spain), with funding from Laboratorios Almirall SA (Barcelona, Spain).

\section{References}

1 Young RR: Spasticity: a review. Neurology 1994;44(11 suppl 9):S12-S20.

$\checkmark 2$ Tan AM, Chakrabarty S, Kimura H, Martin $\mathrm{JH}$ : Selective corticospinal tract injury in the rat induces primary afferent fiber sprouting in the spinal cord and hyperreflexia. J Neurosci 2012;32:12896-12908.

3 Centonze D, Muzio L, Rossi S, Furlan R, Bernardi G, Martino G: The link between inflammation, synaptic transmission and neurodegeneration in multiple sclerosis. Cell Death Differ 2010;17:1083-1091.

4 Baker D, Pryce G, Croxford JL, Brown P, Pertwee RG, Makriyannis A, Khanolkar A, Layward L, Fezza F, Bisogno T, Di Marzo V: Endocannabinoids control spasticity in a multiple sclerosis model. FASEB J 2001;15: 300-302.

5 Hilliard A, Stott C, Wright S, Guy G, Pryce G, Al-Izki S, Bolton C, Giovannoni G: Evaluation of the effects of Sativex (THC BDS: CBD BDS) on inhibition of spasticity in a chronic relapsing experimental allergic autoimmune encephalomyelitis: a model of multiple sclerosis. ISRN Neurol 2012;2012:802649.
6 Gonzalez-Islas C, Garcia-Bereguiain MA, Wenner P: Tonic and transient endocannabinoid regulation of AMPAergic miniature postsynaptic currents and homeostatic plasticity in embryonic motor networks. J Neurosci 2012;32:13597-13607.

7 Rossi S, Furlan R, De Chiara V, Muzio L, Musella A, Motta C, Studer V, Cavasinni F, Bernardi G, Martino G, Cravatt BF, Lutz B, Maccarrone $\mathrm{M}$, Centonze D: Cannabinoid CB1 receptors regulate neuronal TNF- $\alpha$ effects in experimental autoimmune encephalomyelitis. Brain Behav Immun 2011;25:1242-1248.

8 Perez MA, Lungholt BK, Nielsen JB: Shortterm adaptations in spinal cord circuits evoked by repetitive transcranial magnetic stimulation: possible underlying mechanisms. Exp Brain Res 2005;162:202-212.
9 Mori F, Codecà C, Kusayanagi $\mathrm{H}$, Monteleone F, Boffa L, Rimano A, Bernardi G, Koch G, Centonze D: Effects of intermittent theta burst stimulation on spasticity in patients with multiple sclerosis. Eur J Neurol 2010;17: 295-300.

10 Huang YZ, Edwards MJ, Rounis E, Bhatia KP, Rothwell JC: Theta burst stimulation of the human motor cortex. Neuron 2005;45:201206.

11 Koch G, Mori F, Codecà C, Kusayanagi H, Monteleone F, Buttari F, Fiore S, Bernardi G, Centonze D: Cannabis-based treatment induces polarity-reversing plasticity assessed by theta burst stimulation in humans. Brain Stimul 2009;2:229-233.

-12 Rossi S, Bozzali M, Bari M, Mori F, Studer V, Motta C, Buttari F, Cercignani M, Gravina P, Mastrangelo N, Castelli M, Mancino R, Nucci C, Sottile F, Bernardini S, Maccarrone M, Centonze D: Association between a genetic variant of type-1 cannabinoid receptor and inflammatory neurodegeneration in multiple sclerosis. PLoS One 2013;8:e82848. 